\title{
Caracterização do grau de sobrecarga dos cuidadores de utentes dependentes da Unidade de Saúde Familiar USF Descobertas
}

Irene Trindade, ${ }^{1}$ Diogo Almeida, ${ }^{2}$ Margarida Romão, ${ }^{3}$ Sara Rocha, ${ }^{3}$ Sofia Fernandes, ${ }^{2}$ Vasco Varela, ${ }^{1}$ Márcia Braga ${ }^{4}$

\section{RESUMO}

Objetivos: Caracterizar o grau de sobrecarga dos cuidadores de indivíduos dependentes em programa de cuidados domiciliários da Unidade de Saúde Familiar (USF) Descobertas. Correlacionar o grau de sobrecarga com o grau de dependência e identificar as dimensões do bem-estar do cuidador mais afetadas.

Tipo de estudo: Observacional transversal analítico.

Local: USF Descobertas.

População: Cuidadores informais de indivíduos dependentes em cuidados domiciliários médicos ou enfermagem da USF Descobertas, entre janeiro e fevereiro de 2015.

Métodos: Foram incluídos todos os cuidadores informais de indivíduos dependentes em programa de cuidados domiciliários médicos ou de enfermagem realizados durante dois meses. Foram aplicados telefonicamente três questionários: um para a caracterização sociodemográfica do cuidador, a escala de Katz ao indivíduo dependente e a escala de Zarit ao cuidador. Foram utilizados o coeficiente de Spearman e o teste de Kruskal-Wallis para a análise dos dados.

Resultados: Foram identificados 174 dependentes, 65 dos quais cumpriam os critérios de inclusão e corresponderam a 60 cuidadores. Cinco cuidadores prestavam assistência a dois dependentes simultaneamente. Em relação à escala de Zarit, $44,6 \%$ $(n=29)$ dos cuidadores apresentou sobrecarga intensa, $20,0 \%(n=13)$ sobrecarga ligeira e $35,4 \%(n=23)$ não apresentou sobrecarga. A média do valor da escala de Katz foi de 2,78. Não se estabeleceu relação estatisticamente significativa entre o grau de sobrecarga do cuidador e o nível de dependência $(p=0,053)$.

Conclusões: A maioria dos cuidadores apresentou sobrecarga, sobretudo a nível intenso. Os resultados obtidos corroboraram os dados apresentados noutros estudos nacionais. A avaliação sistemática da sobrecarga do cuidador e mobilização dos recursos adequados constituem intervenções a incluir nos planos realizados pelos profissionais de saúde. Perante a escassez de estudos neste domínio considerou-se importante a futura realização de estudos numa população de maior dimensão.

Palavras-chave: Cuidador; Sobrecarga; Dependente; Cuidados domiciliários; Zarit; Cuidados paliativos.

\section{INTRODUÇÃO}

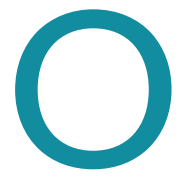

envelhecimento da população aliado ao aumento do número de indivíduos com doença crónica e incapacidade exige medidas de prestação de cuidados de saúde dirigidas aos indivíduos dependentes e seus cuidadores. Segundo o EUROSTAT, a taxa de indivíduos acima dos 65 anos de idade na população europeia atingiu
17,9\% em 2012. ${ }^{1}$ Em Portugal, 19\% da população tem 65 ou mais anos de idade. ${ }^{2}$ Mais de metade da popula-

1. Médico Interno de Medicina Geral e Familiar ( $3^{\circ}$ Ano). USF Descobertas, ACeS Lisboa Ocidental de Oeiras

2. Médico Interno de Medicina Geral e Familiar ( $2^{\circ}$ Ano). USF Descobertas, ACeS Lisboa Ocidental de Oeiras

3. Médico Interno de Medicina Geral e Familiar ( $4^{\circ}$ Ano). USF Descobertas, ACeS Lisboa Ocidental de Oeiras

4. Médica Assistente de Medicina Geral e Familiar. USF Descobertas, ACeS Lisboa Ocidental de Oeiras 
ção portuguesa tem uma ou mais doenças crónicas $(52,3 \%)$ e cerca de um quarto $(26,5 \%)$ tem pelo menos duas doenças crónicas. ${ }^{3}$ A tendência de aumento do número de indivíduos dependentes e consequente necessidade de apoio no domicílio determina a importância dos cuidados domiciliários em cuidados de saúde primários (CSP). ${ }^{4}$

A Unidade de Saúde Familiar (USF) Descobertas, em Lisboa, apresenta um total de 16.300 utentes; destes, $27 \%$ tem mais de 65 anos de idade. Nos dois primeiros meses do ano de 2015174 utentes encontravam-se em programa de cuidados de saúde domiciliários.

Dependência é a situação em que se encontra a pessoa que, por falta ou perda de autonomia física, psíquica ou intelectual, resultante ou agravada por outras comorbilidades, não consegue, por si só, realizar as atividades de vida diária. ${ }^{6}$ Paralelamente ao conceito de indivíduo dependente, considera-se cuidador aquele que presta cuidados informais, que consistem na ajuda ou supervisão prestada às pessoas em situação de dependência. Geralmente este tipo de cuidados não é remunerado e é prestado pela família, amigos ou vizinhos. ${ }^{6}$ Estes cuidados informais podem envolver as componentes físicas, psicológicas e financeiras dos cuidadores de uma forma considerável. ${ }^{7} \mathrm{O}$ bem-estar dos cuidadores de indivíduos dependentes pode ser avaliado em quatro dimensões: participação social, recursos financeiros, saúde física e mental. ${ }^{8-9}$ Esta situação de acumulação de tarefas coloca o cuidador em risco de sobrecarga. O número de horas por dia envolvidas na prestação de cuidados, o recurso a suporte emocional, funcional e físico são preditores de sobrecarga do cuidador. ${ }^{10}$ As características da situação em que os cuidados são prestados relacionam-se mais estreitamente com o bem-estar dos cuidadores do que as características atribuíveis à doença do indivíduo alvo de cuidados. ${ }^{8}$ Para além destas contingências é frequente os cuidadores negligenciarem a sua própria saúde e ocultarem informação acerca dos seus próprios problemas de saúde. ${ }^{11}$

Paralelamente à sobrecarga existe uma vertente que aponta para benefícios e ganhos do cuidador como a sensação de maior utilidade, aprendizagem de novas aptidões, atribuição de significado ao próprio, cumprimento de um dever e relação de companheirismo com o indivíduo alvo de cuidados. ${ }^{12-16}$
O apoio social pode ter um efeito atenuante da sobrecarga do cuidador. ${ }^{17-22}$

Diversos estudos internacionais que avaliam o grau de sobrecarga dos cuidadores de doentes dependentes e caracterizaram o perfil dos cuidadores revelam níveis elevados de sobrecarga. ${ }^{8,2-21}$ Em Portugal existem estudos na área dos cuidados paliativos, onde a população dependente apresenta doença oncológica em fase avançada. ${ }^{23-24}$ Outros estudos nacionais semelhantes abrangem outras populações específicas de indivíduos dependentes, nomeadamente com demência. ${ }^{25-26}$ Não existem publicados estudos nacionais sobre o grau de sobrecarga dos cuidadores de indivíduos dependentes em geral e seguidos em cuidados de saúde primários, ou seja, de dependentes cuja natureza da incapacidade não se encontra limitada a uma entidade nosológica específica, como a doença oncológica ou a demência.

O objetivo primário deste trabalho é caracterizar o grau de sobrecarga dos cuidadores de indivíduos dependentes em programa de cuidados domiciliários da USF Descobertas. Secundariamente, pretende-se correlacionar esta avaliação com o grau de dependência do indivíduo alvo de cuidados e identificar as dimensões do bem-estar do cuidador mais afetadas pela sobrecarga.

\section{MÉTODOS}

Para a realização do trabalho optou-se por um desenho de estudo observacional transversal analítico.

Critérios de inclusão: todos os adultos cuidadores informais de indivíduos dependentes em programa de cuidados domiciliários médicos ou de enfermagem na USF Descobertas durante o período de janeiro e fevereiro de 2015. Nos casos em que o dependente recebeu apoio domiciliário institucional, o cuidador que articulou a prestação desse serviço foi incluído no estudo. Foram incluídos, ainda, os casos em que se identificou mais do que um cuidador e as tarefas foram equitativamente distribuídas entre eles.

Critérios de exclusão: todos os cuidadores de indivíduos dependentes falecidos à data de recolha dos dados, cuidadores de recém-nascidos, recém-nascidos e cuidadores remunerados. Foi recolhida informação acerca dos motivos de exclusão dos indivíduos não elegíveis para o estudo. 
A recolha de dados foi realizada a toda a população alvo através da aplicação de um questionário por via telefónica. Este questionário foi aplicado após a obtenção do consentimento informado oral contemplado no protocolo de investigação, com parecer positivo da Comissão de Ética para a Saúde da Administração Regional de Saúde de Lisboa e Vale do Tejo. A recolha de dados foi realizada por entrevistadores médicos do primeiro, segundo e quarto anos do internato complementar de medicina geral e familiar da USF Descobertas. O questionário aplicado encontrava-se dividido em três partes. A primeira dirigida à caracterização sociodemográfica do cuidador, da sua atividade como cuidador e eventuais apoios utilizados. Na segunda parte aplicou-se a escala de Katz para avaliação do nível de dependência do indivíduo dependente. Finalmente, para a avaliação do grau de sobrecarga do cuidador utilizou-se o questionário Zarit Burden Interview, com autorização do autor do artigo que validou essa mesma ferramenta para a população portuguesa. ${ }^{27} \mathrm{~A}$ escala de Katz foi originalmente descrita e validada por Katz e colaboradores em $1963 .{ }^{5}$ Lino e colaboradores validaram esta mesma escala para a língua portuguesa, mantendo os mesmos seis itens originais. $\mathrm{O}$ resultado final da aplicação da escala de Katz corresponde ao somatório da pontuação atribuída à capacidade de desempenho de cada uma das seis categorias de atividades de vida diária. O indivíduo pode ser considerado totalmente dependente - zero pontos; ou independente - seis pontos. Às pontuações intermédias correspondem três níveis de dependência: ligeira (cinco pontos), moderada (3-4 pontos) e grave (1-2 pontos). ${ }^{28}$

Para a avaliação da sobrecarga do cuidador utilizouse a escala de Zarit validada para a língua portuguesa, em 2010, por Sequeira. ${ }^{27}$ Este autor utilizou a última versão da Escala de Zarit, reduzida de 29 para 22 itens, validada por Bédard e colaboradores em 2001. Cada item desta escala pode ser caracterizado qualitativamente pelo cuidador como: «nunca», «quase nunca», «às vezes», «muitas vezes» ou «quase sempre»e, para a análise de dados, o investigador atribui a pontuação 1,2 , $3,4,5$ a cada uma das expressões anteriores, respetivamente. Assim, a pontuação total pode variar entre $22 \mathrm{e}$ 110 - quanto mais elevado o valor, maior a perceção de sobrecarga pelo cuidador. Foi convencionado que uma pontuação total superior a 56 corresponde a sobrecar- ga intensa, entre 46 e 56 a sobrecarga ligeira e inferior a 46 à ausência de sobrecarga. ${ }^{12,27,29}$ Nos casos em que o mesmo cuidador prestava cuidados a dois indivíduos dependentes diferentes, o cuidador respondeu a duas séries de questionários, respetivamente.

A análise estatística dos dados foi efetuada através da avaliação do coeficiente de Spearman e do teste de Kruskal-Wallis, através do programa Statistical Package for the Social Sciences (SPSS), versão 21.

\section{RESULTADOS}

Durante janeiro e fevereiro de 2015 foram realizadas visitas domiciliárias a 174 indivíduos inscritos na USF Descobertas. Destes 174 indivíduos elegíveis excluíram-se 74 após aplicação dos critérios de exclusão. Foram, ainda, identificados 35 casos de impossibilidade de aplicação dos inquéritos aos cuidadores, nomeadamente por cuidador incontactável $(n=14)$, por dependente institucionalizado $(n=14)$, por recusa na resposta ao questionário $(n=5)$ e por transferência do dependente para outra USF ( $n=2)$. Obtiveram-se, assim, 65 indivíduos dependentes e 60 cuidadores. Destes cuidadores cinco prestavam cuidados a dois indivíduos dependentes. Foram aplicados 65 questionários correspondentes aos 65 indivíduos dependentes.

\section{Cuidadores}

A idade média da população de cuidadores $(n=60)$ estudada foi de 64,2 anos e o tempo médio de duração do período de prestação de cuidados foi de 8,6 anos. Os restantes dados sociodemográficos encontram-se resumidos no Quadro I.

Relativamente ao grau de sobrecarga da população de cuidadores entrevistados, 44,6\% ( $n=29$ ) apresentou sobrecarga intensa, $20,0 \%(n=13)$ sobrecarga ligeira e $35,4 \%$ ( $n=23)$ não apresentou sobrecarga (Figura 1).

Os resultados relativos à distribuição da média $\mathrm{e}$ moda de pontuações atribuídas a cada item da escala de Zarit encontram-se ilustrados na Figura 2. Os itens mais pontuados foram por ordem decrescente de grau de sobrecarga: o item 8 ( $«$ Considera que o seu familiar está dependente de si?»), o item 14 («Acredita que o seu familiar espera que você cuide dele como se fosse a única pessoa com quem ele(a) pudesse contar?»), o item 7 («Tem receio pelo futuro destinado ao seu familiar?»), o item 2 («Considera que devido ao tempo que dedica 
ao seu familiar já não dispõe de tempo suficiente para as suas tarefas?»), o item 15 («Considera que não dispõe de economias suficientes para cuidar do seu familiar e para o resto das despesas que tem?»), o item 3 («Sente-se tenso/a quando tem de cuidar do seu familiar e ainda tem outras tarefas por fazer?») e o item 22 («Em geral sente-se muito sobrecarregado por ter de cuidar do seu familiar?»). Os itens que apresentaram menor pontuação, por ordem crescente de sobrecarga, foram: o item 4 («Sente-se envergonhado(a) pelo comportamento do seu familiar?»), o item 1 ( Sente que o seu familiar solicita mais ajuda do que aquela que realmente necessita?»), o item 5 («Sente-se irritado/a quando está junto do seu familiar?»), o item 20 ( Sente que poderia fazer mais pelo seu familiar?») e o item 13 («Sente-se pouco à vontade em convidar amigos para o(a) visitarem devido ao seu familiar?»).

\section{Indivíduos dependentes}

Relativamente aos indivíduos dependentes incluídos no estudo ( $n=65), 66,2 \%$ $(n=43)$ eram do sexo feminino e $33,8 \%(n=22)$ do sexo masculino. Os restantes dados relativos à caracterização etária e ao nível de dependência (Escala de Katz) encontram-se resumidos no Quadro II.

\section{Análise estatística}

Não se obteve associação estatisticamente significativa entre o grau de sobrecarga do cuidador e as seguin-

\begin{tabular}{|c|c|c|c|}
\hline \multirow{2}{*}{ Categoria de dados } & \multirow{2}{*}{ Variáveis } & \multicolumn{2}{|c|}{ Frequência } \\
\hline & & Absoluta (n) & Relativa (\%) \\
\hline \multirow[t]{3}{*}{ Género } & Feminino & 51 & 78,5 \\
\hline & Masculino & 14 & 21,5 \\
\hline & Total & 65 & 100,0 \\
\hline \multirow[t]{5}{*}{ Estado civil } & Solteiro & 10 & 15,4 \\
\hline & Casado & 32 & 49,2 \\
\hline & Divorciado & 13 & 20,0 \\
\hline & Outro & 10 & 15,4 \\
\hline & Total & 65 & 100,0 \\
\hline \multirow[t]{5}{*}{ Escolaridade } & Analfabetismo & 1 & 1,5 \\
\hline & $1^{\circ}$ ao $3^{\circ}$ ciclo & 35 & 53,9 \\
\hline & Ensino secundário & 16 & 24,6 \\
\hline & Ensino superior & 13 & 20,0 \\
\hline & Total & 65 & 100,0 \\
\hline \multirow{7}{*}{$\begin{array}{l}\text { Grau de parentesco em } \\
\text { relação ao indivíduo } \\
\text { dependente }\end{array}$} & Filho & 34 & 52,3 \\
\hline & Cônjuge & 13 & 20,0 \\
\hline & Sobrinho & 1 & 1,5 \\
\hline & Irmão & 2 & 3,1 \\
\hline & Vizinho & 3 & 4,6 \\
\hline & Outro & 12 & 18,5 \\
\hline & Total & 65 & 100,0 \\
\hline \multirow{3}{*}{$\begin{array}{l}\text { Experiência anterior } \\
\text { como cuidador }\end{array}$} & Sem experiência anterior & 47 & 72,3 \\
\hline & Com experiência anterior & 18 & 27,7 \\
\hline & Total & 65 & 100,0 \\
\hline \multirow[t]{3}{*}{ Situação profissional } & Ativo & 15 & 23,0 \\
\hline & Inativo (desempregado/reformado) & 50 & 77,0 \\
\hline & Total & 65 & 100,0 \\
\hline \multirow{3}{*}{$\begin{array}{l}\text { Existência de outros } \\
\text { cuidadores }\end{array}$} & Sim & 30 & 46,2 \\
\hline & Não & 35 & 53,8 \\
\hline & Total & 65 & 100,0 \\
\hline
\end{tabular}

tes variáveis: idade do dependente $(p=0,181)$, idade do cuidador $(p=0,622)$, duração da prestação de cuidados $(p=0,464)$ e o nível de dependência avaliado pela escala de $\operatorname{Katz}(p=0,053)$.

\section{DISCUSSÃO}

Neste trabalho verificou-se que a maioria dos cuidadores estudados apresentou sobrecarga (65\% do total dos cuidadores questionados) e que esta foi predo- 


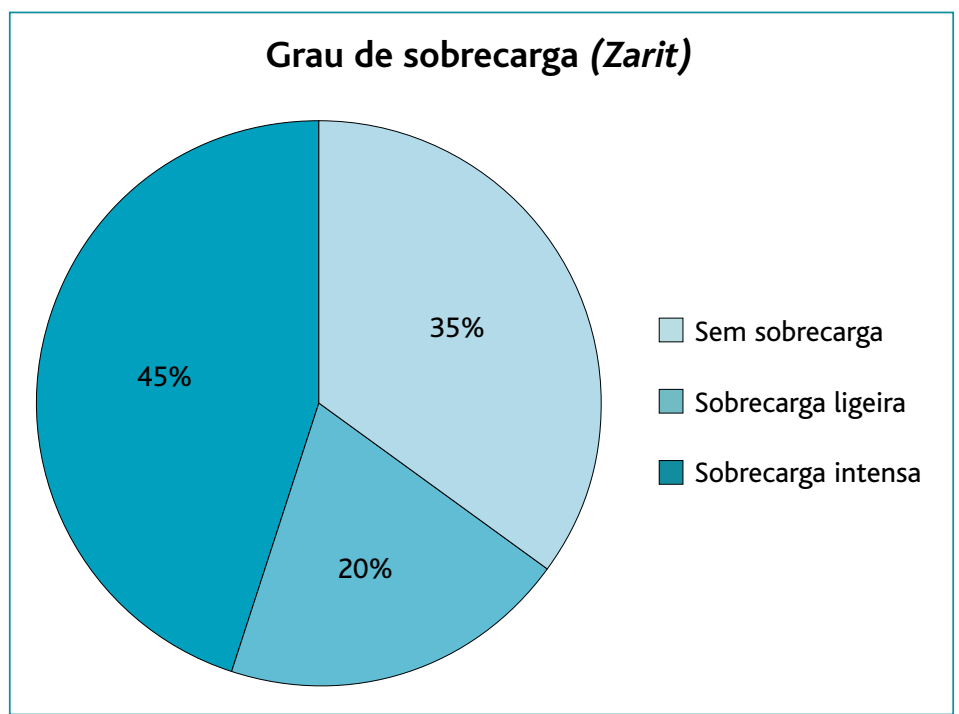

Figura 1. Distribuição do grau de sobrecarga da população de cuidadores em estudo através da aplicação da escala de Zarit.

minantemente intensa $(69,2 \%$ dos cuidadores identificados com sobrecarga). A dimensão do bem-estar do cuidador mais afetada foi a das «expectativas face ao cuidar", uma vez que os itens a ela pertencentes $-7,8$, 14 e 15 - corresponderam, respetivamente, ao terceiro, primeiro, segundo e quinto mais pontuados ( $c f$. Gráfico 2). Seguiu-se, como segundo domínio mais afetado, 0 «impacto na prestação de cuidados» através dos itens 2,3 e 22. Tais resultados aproximaram-se dos obtidos por Sequeira e colaboradores no processo de validação da escala de Zarit para a população portuguesa. Para este autor, os dois itens mais pontuados por cuidadores de idosos sem demência foram os mesmos que os obtidos neste trabalho (itens 8 e 14)..$^{30-31}$

Relativamente às características dos cuidadores estudados tratou-se de uma população de idade avançada, na sua maioria composta por mulheres casadas, com um baixo grau de escolaridade e sem atividade profissional, que eram filhas ou esposas dos dependentes. Estes dados foram sobreponíveis aos de outros estudos nacionais. ${ }^{30-31}$ Tal poderá ser justificado pelo papel da mulher na sociedade ao longo dos últimos séculos, nomeadamente ao nível da prestação de cuidados à família. ${ }^{32}$

No que respeita às características dos indivíduos dependentes, os resultados obtidos foram também ao en-

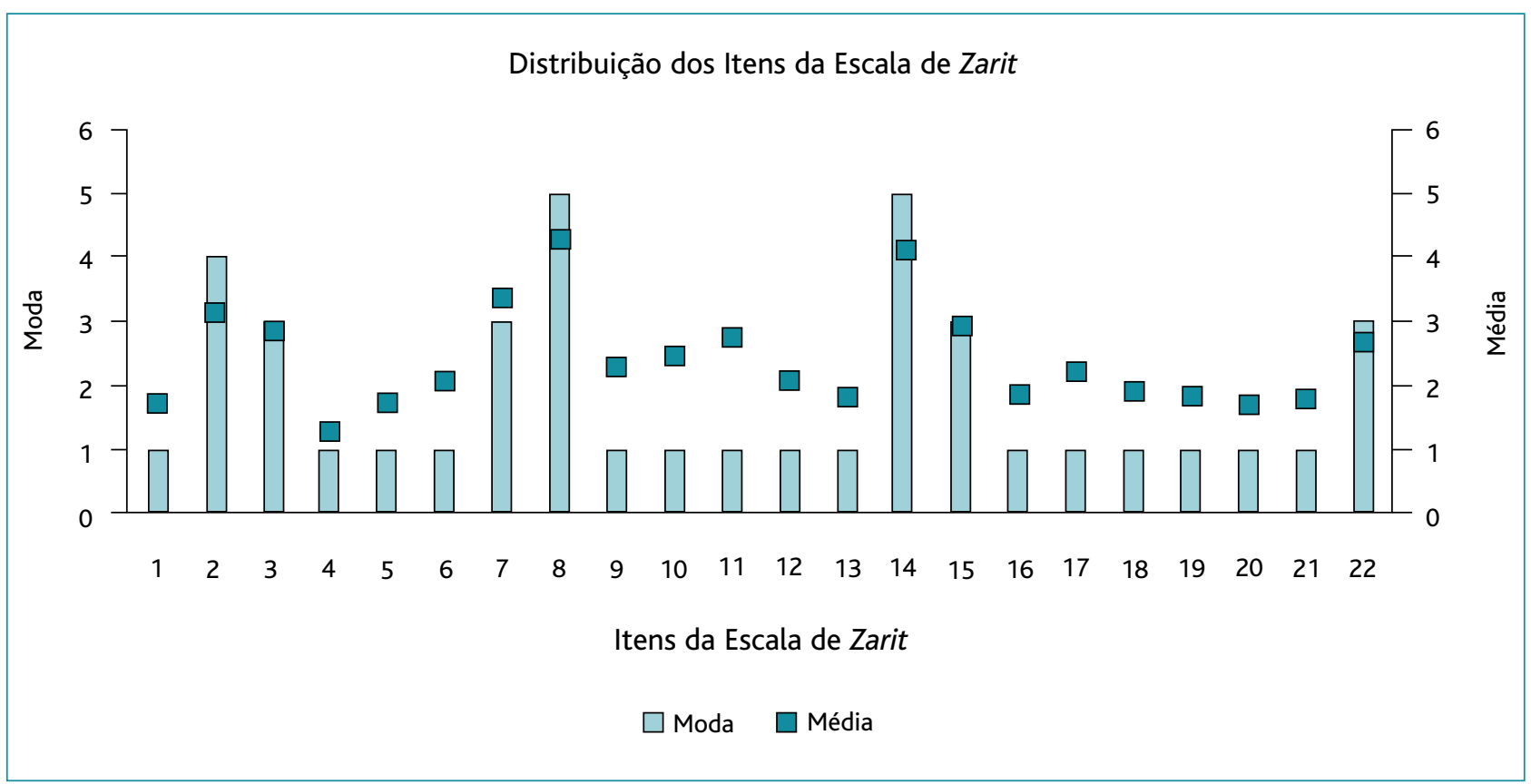

Figura 2. Distribuição da pontuação média e moda atribuída a cada item da escala de Zarit. 
QUADRO II. Dados etários e sobre o grau de

dependência dos indivíduos alvo de cuidados

\begin{tabular}{l|c|c} 
Variáveis & $\begin{array}{c}\text { Idade do indivíduo } \\
\text { dependente (anos) }\end{array}$ & Escala de Katz \\
\hline Média & 84,8 & 2,78 \\
Mediana & 87,0 & 3,00 \\
Valor máximo & 104 & 6 \\
Valor mínimo & 37 & 0 \\
\hline
\end{tabular}

contro dos de outros estudos portugueses. ${ }^{30-31}$ A maioria corresponde a indivíduos do sexo feminino de idade avançada, o que poderá ser fruto do envelhecimento da população e do aumento da esperança média de vida das mulheres comparativamente à dos homens. ${ }^{2,33}$

Contudo, no presente trabalho, os níveis de dependência foram inferiores aos determinados para outras realidades nacionais. ${ }^{30-31}$ Tal poderá dever-se ao facto de contrariamente aos outros estudos, a dependência para atividades instrumentais não ter sido avaliada, uma vez que apenas se recorreu à Escala de Katz. ${ }^{30-31}$ Identificaram-se indivíduos em programa de cuidados domiciliários da USF cuja inscrição é justificada não só pela sua mobilidade reduzida como por barreiras arquitetónicas do seu próprio local de residência. Nos casos em que não apresentavam limitação para a realização das atividades de vida diária mostravam dependência para as atividades instrumentais.

No que respeita à duração média da prestação de cuidados (8,6 anos), esta foi significativamente superior à obtida na maioria dos estudos (cinco anos). ${ }^{30-31}$

Tal como nos trabalhos de Marques, Custódio e seus colaboradores, os autores também não verificaram relação entre o grau de sobrecarga e a duração da prestação de cuidados $(p=0,464)$, a idade do cuidador $(p=0,622)$ e a idade do dependente $(p=0,181) .^{34-35}$

De acordo com outros estudos nacionais, o nível de dependência foi determinante para o grau de sobrecarga do cuidador. A sobrecarga subjetiva - designadamente ao nível das expectativas face ao cuidar - foi tanto mais elevada quanto maior a dependência. ${ }^{30-31} \mathrm{Nes}$ te trabalho, o valor de $p$ para a associação entre o grau de dependência avaliado pela escala de Katz e o nível de sobrecarga do cuidador foi de 0,053, próximo do limiar para se considerar uma associação estatistica- mente significativa. Tanto a dimensão da população como a avaliação da dependência exclusivamente dirigida às atividades básicas de vida diária poderão ter limitado a obtenção da associação verificada por outros autores.

Este trabalho apresentou ainda outras limitações, nomeadamente as relacionadas com a seleção da população-alvo e com o elevado número de dependentes e cuidadores não incluídos no estudo.

Uma vez que a seleção da população decorreu da identificação dos indivíduos em programa de cuidados domiciliários numa USF, não foram avaliados outros potenciais cuidadores de dependentes que poderiam não estar abrangidos por esse programa. A magnitude deste viés é inestimável, mas pode presumir-se que decorrerá no sentido da sobrestimação do grau de sobrecarga. Esta presunção decorre de dois pressupostos: por um lado, de que existe uma real associação entre o grau de sobrecarga e grau de dependência e, por outro, que os utentes capazes de se deslocar à USF poderão apresentar menor necessidade de cuidados e, por inerência, induzir menores graus de sobrecarga nos cuidadores.

Neste estudo não foram avaliados 109 de 174 indivíduos em programa de cuidados domiciliários no período estabelecido, a maioria por apresentar critérios de exclusão. Contudo, existiram 35 casos que não se enquadraram nos critérios de exclusão e que poderiam ter alterado os resultados obtidos. Estes corresponderam à soma dos 14 cuidadores incontactáveis, 14 utentes dependentes institucionalizados, cinco cuidadores que recusaram participar no estudo e dois utentes que foram transferidos da unidade.

Este trabalho engloba cuidadores informais de indivíduos sem idade ou patologias específicas, constituindo, assim, uma população heterogénea. Pelo contrário, a maioria dos estudos publicados nesta área reportou a indivíduos idosos ou com patologia demencial, pelo que os dados aqui apresentados poderão ser mais representativos da realidade a nível dos cuidados de saúde primários.

Perante a evidência de que a maioria dos cuidadores de indivíduos dependentes em programa de cuidados domiciliários da USF apresentou sobrecarga, os autores consideram que a sua avaliação sistemática poderá constituir um elemento-chave na prática clínica. 
Esta deverá ser ativamente realizada pelo médico ou enfermeiro de família e registada adequadamente no processo clínico de forma a identificar os cuidadores em risco e a monitorizar a evolução dos seus níveis de sobrecarga.

Os autores consideram, ainda, que para cada situação de dependência seja feito um levantamento dos recursos de que cada cuidador poderá beneficiar (quer a nível familiar quer a nível institucional), procurandose estabelecer áreas de melhoria. Para este efeito, o uso do ecomapa, ou outros instrumentos de avaliação, e a promoção de reuniões conjuntas com representantes do serviço social poderão ser úteis na elaboração do plano dirigido à prevenção da sobrecarga de cada cuidador.

As equipas de família deverão, pois, ser conhecedoras dos recursos disponíveis na comunidade (apoios domiciliários, teleassistência, centros de dia, ajudas de custo, rede nacional de cuidados continuados integrados), sendo que para esse efeito uma abordagem interdisciplinar entre médico, enfermeiro, administrativo, assistente social e psicólogo poderá revelar-se uma mais-valia.

\section{CONCLUSÃO}

Dado que a maioria dos cuidadores de indivíduos dependentes em programa de cuidados domiciliários da USF Descobertas apresentou sobrecarga, a avaliação sistemática da sobrecarga do cuidador poderá constituir um elemento-chave na prática de cuidados domiciliários. A disponibilidade de tempo do cuidador e a insuficiência económica foram as dimensões do bem-estar mais afetadas pela sobrecarga. A abordagem interdisciplinar - médico, enfermeiro, assistente social, psicólogo, administrativo, entre outros intervenientes - poderá ser a resposta mais adequada para a melhoria do grau de sobrecarga e dos cuidados domiciliários prestados ao grupo populacional vulnerável em estudo. Sendo as dimensões psicossociais e financeiras as mais afetadas nesta população tem-se a oportunidade de dirigir a disponibilização de recursos da comunidade e a adaptação da abordagem holística dos cuidadores individualmente, de modo personalizado.

Perante a escassez de estudos sobre a associação entre as escalas de Zarit e Katz, e dado os resultados obtidos serem próximos de valores estatisticamente sig- nificativos, os autores consideram importante a realização futura de novos estudos com uma população de maiores dimensões. Futuramente seria interessante identificar cuidadores de indivíduos não integrados em programa de cuidados domiciliários, mas simultaneamente dependentes, de forma a sinalizar um maior número de casos de cuidadores em risco de sobrecarga.

\section{AGRADECIMENTOS}

A toda a equipa da USF Descobertas. A todos os utentes e cuidadores que aceitaram participar no presente estudo.

\section{REFERÊNCIAS BIBLIOGRÁFICAS}

1. EUROSTAT. Population structure and ageing [Internet]. Luxembourg: Publications Office of the European Union; 2014 May [updated 2016 Dec 22]. Available from: http://ec.europa.eu/eurostat/statistics-explained/index.php/Population_structure_and_ageing

2. Instituto Nacional de Estatística. Censos 2011. Lisboa: INE; 2014.

3. Instituto Nacional de Estatística, Instituto Nacional de Saúde Dr Ricardo Jorge. Inquérito Nacional de Saúde 2005/2006 [Internet]. Lisboa: INE/INSA; 2009. Available from: http://www2.insa.pt/sites/INSA/Portugues/Publicacoes/Outros/Documents/Epidemiologia/INS_05_06.pdf

4. Maayan N, Soares-Weiser K, Lee H. Respite care for people with dementia and their carers. Cochrane Database Syst Rev. 2014;(1):CD004396.

5. Katz S, Ford AB, Moskowitz RW, Jackson BA, Jaffe MW. Studies of illness in the aged. The index of ADL: a standardized measure of biological and psychosocial function. JAMA. 1963;185(12):914-9.

6. Unidade de Missão para os Cuidados Continuados Integrados. Glossário - Rede nacional de cuidados continuados integrados [Internet]. Lisboa: ACSS; 2009. Available from: http://www.acss.min-saude.pt/wp-content/uploads/2016/07/Glossario-da-RNCCI.pdf

7. Candy B, Jones L, Drake R, Leurent B, King M. Interventions for supporting informal caregivers of patients in the terminal phase of a disease. Cochrane Database Syst Rev. 2011;(6):CD007617.

8. George LK, Gwyther LP. Caregiver well-being: a multidimensional examination of family caregivers of demented adults. Gerontologist. 1986;26(3):253-9.

9. Parker D, Mills S, Abbey J. Effectiveness of interventions that assist caregivers to support people with dementia living in the community: a systematic review. Int J Evid Based Healthc. 2008;6(2):137-72.

10. Chang HY, Chiou CJ, Chen NS. Impact of mental health and care giver burden on family caregivers' physical health. Arch Gerontol Geriatr. 2010;50(3):267-71.

11. Ward-Griffin C, McKeever P. Relationships between nurses and family caregivers: partners in care? ANS Adv Nurs Sci. 2000;22(3):89-103.

12. Ferreira F, Pinto A, Laranjeira A, Pinto AC, Lopes A, Viana A, et al. Validação da escala de Zarit: sobrecarga do cuidador em cuidados paliativos domiciliários, para população portuguesa [Validation of the Zarit's scale ("Zarit Burden Interview") for the Portuguese population in the field of domiciliary palliative patient care]. Cadernos Saúde. 2010;3(2):13-9. Portuguese

13. Koerner SS, Kenyon DB, Shirai Y. Caregiving for elder relatives: which caregivers experience personal benefits/gains? Arch Gerontol Geriatr. 
2009 Mar-Apr;48(2):238-45.

14. Lawton MP, Moss M, Kleban MH, Glicksman A, Rovine M. A two factor model of caregiving appraisal and psychological well-being. J Gerontol. 1991;46(4):P181-9.

15. Beach SR, Schulz R, Yee JL, Jackson S. Negative and positive health effects of caring for a disabled spouse: longitudinal findings from the caregiver health effects study. Psychol Aging. 2000;15(2):259-71.

16. Baronet A. The impact of family relations on caregivers' positive and negative appraisal of their caregiving activities. Fam Relat. 2003;52:13742.

17. Cohen CA, Colantonio A, Vernich L. Positive aspects of caregiving: rounding out the caregiver experience. Int J Geriatr Psychiatry. 2002;17(2):184-8

18. Song LY, Biegel DE, Milligan SE. Predictors of depressive symptomatology among lower social class caregivers of persons with chronic mental illness. Community Ment Health J. 1997;33(4):269-86.

19. Pohl JM, Given CW, Collins CE, Given BA. Caregiving in daughters and daughters-in-law caring for disabled aging parents. Health Care Women Int. 1994;15(5):385-95.

20. Li LW, Seltzer MM, Greenberg JS. Social support and depressive symptoms: differential patterns in wife and daughter caregivers. J Gerontol B Psychol Sci Soc Sci. 1997;52(4):S200-11.

21. Sander AM, High WM Jr, Hannay HJ, Sherer M. Predictors of psycho logical health in caregivers of patients with closed head injury. Brain Inj. 1997;11(4):235-49.

22. Edwards NE, Scheetz PS. Predictors of burden for caregivers of patients with Parkinson's disease. J Neurosci Nurs. 2002;34(4):184-90.

23. Fernandes JJ. Cuidar no domicílio: a sobrecarga do cuidador familiar [Dissertation]. Lisboa: Faculdade de Medicina, Universidade de Lisboa; 2009.

24. Delalibera M, Presa J, Barbosa A, Leal I. Sobrecarga no cuidar e suas repercussões nos cuidadores de pacientes em fim de vida: revisão sistemática da literatura [Burden of caregiving and its repercussions on caregivers of end-of-life patients: a systematic review of the literature]. Ciênc Saúde Colet. 2015;20(9):2731-47. Portuguese

25. Ferreira IN. A sobrecarga do cuidador informal de pessoas com dependência [Dissertation]. Setúbal: Escola Superior de Saúde, Instituto Politécnico de Setúbal; 2013.

26. Pera LF. Avaliação das dificuldades e sobrecarga do cuidador informal de idosos dependentes [Dissertation]. Bragança: Escola Superior da Saúde, Instituto Politécnico de Bragança; 2012.

27. Sequeira CA.Adaptação e validação da Escala de Sobrecarga do Cuidador de Zarit [Adaptation and validation of Zarit Burden Interview Scale]. Rev Referência. 2010;॥(12):9-16. Portuguese

28. Lino VT, Pereira SR, Camacho LA, Ribeiro Filho ST, Buksman S. Adaptação transcultural da escala de independência em Atividades da Vida Diária (Escala de Katz) [Cross-cultural adaptation of the Independence in Activities of Daily Living Index (Katz Index)]. Cad Saúde Pública. 2008;24(1):103-12. Portuguese

29. Bédard M, Molloy DW, Squire L, Dubois S, Lever JA, O'Donnell M. The Zarit Burden Interview: a new short version and screening version. Gerontologist. 2001;41(5):652-7.

30. Cordeiro LA. Cuidador informal de idosos dependentes: dificuldades e sobrecarga [Dissertation]. Viseu: Escola Superior de Saúde, Instituto Politécnico de Viseu; 2011.

31. Ricarte LF. Sobrecarga do cuidador informal de idosos dependentes no Concelho da Ribeira Grande [Dissertation]. Porto: Instituto de Ciências Biomédicas de Abel Salazar, Universidade do Porto; 2009.

32. Barnett RC. Women and work: where are we, where did we come from, and where are we going? [Preface]. J Soc Issues. 2004;60(4):667-74.

33. Carrilho MJ, Craveiro ML. A situação demográfica recente em Portugal [Demographic changes in Portugal]. Rev Estudos Demográficos. 2015;(54):57-107. Portuguese

34. Marques S. Os cuidadores informais de doentes com acidente vascular cerebral. Interacções. 2005;(9):130-41.

35. Custódio JR. Sobrecarga e estratégias de coping do cuidador informal do idoso dependente [Dissertation]. Coimbra: Instituto Superior Miguel Torga; 2011.

\section{CONFLITO DE INTERESSES}

Os autores declaram não ter conflitos de interesses.

\section{COMISSÃO DE ÉTICA}

Estudo realizado após parecer favorável da Comissão de Ética para a Saúde da ARSLVT.

\section{ENDEREÇO PARA CORRESPONDÊNCIA}

Irene Isabel Donat Trindade

R. Fernão Mendes Pinto, 19 - 1400-279 Lisboa

E-mail: trindade.irene@gmail.com

Recebido em 08-05-2016

Aceite para publicação em 22-02-2017 


\section{ABSTRACT}

\section{CAREGIVER BURDEN WITH COMPLEX PATIENTS IN A HOME CARE PROGRAM}

Objectives: To characterize caregiver burden in the care of dependent individuals in the home care program of the Descobertas Family Health Unit, to test the association between caregiver burden and the level of dependence of the patient, and to identify the dimensions of caregiver wellbeing that were affected.

Study design: Observational study.

Location: USF Descobertas.

Population: Informal caregivers of dependent individuals in a home care program.

Methods: Each informal caregiver of a dependent individual in the medical and nursing home care program was included in the study over a two-month period. We conducted interviews by telephone using three questionnaires: a social and demographic description of the caregivers, the Katz scale to evaluate the level of dependency of the patient, and the Zarit caregiver burden interview. Spearman coefficients and Kruskal-Wallis tests were used to test for associations between variables.

Results: A total of 174 dependent patients were identified, 65 of whom fulfilled the inclusion criteria and who were cared for by 60 caregivers. Five caregivers were taking care of two dependents simultaneously. Regarding the Zarit scale, 44.6\% ( $n=29$ ) of the caregivers reported a heavy burden, $20.0 \%(n=13)$ a light burden and $35.4 \%(n=23)$ no burden. The mean value of the Katz scale was 2.78. There was no statistically significant correlation between caregiver burden and the level of dependency of the patient $(p=0.053)$.

Conclusion: The burden of caregiving was identified in the majority of informal caregivers and was reported to be at an intense level. The items identified on the Zarit Scale were similar to those found in other national studies. Healthcare professionals should perform a systematic evaluation of caregiver burden and be able to intervene when needed. Further study of this issue is required in larger populations.

Keywords: Caregiver; Burden; Dependent; Home care services; Zarit; Palliative care. 